\title{
Acoustic Energy Harvesting Through MM- Piezoelectric Harvester Model
}

\author{
M. Sreenivasulu, V. Usha Shree, P. Chandrasekhar Reddy
}

\begin{abstract}
In recent years use of electronics devices such as electronic communication device and wireless sensor network are increasing rapidly since they consume low power, Furthermore increase in energy consumption has led researcher to focus on environmental energy utilization as it possesses several advantage in comparison with electrochemical battery. Moreover harvesting of environmental energy includes from raindrops, water current, and wind flow, thermal and solar has been few research area which is focused widely. In past several researcher focused on energy harvesting however these method has two main disadvantage either their material volume is large to adopt for real time or they obtain very low output power. Hence In this paper we focus on energy harvesting based on acoustic; further to achieve the optimal energy harvesting we propose a mechanism named MM-PEH(Multilayered multiple Piezoelectric Harvester) we use lead zircon ate titnate as a piezoelectric generator; this cantilever type is used for harvesting sound energy from various loudspeaker with fair distance. Here we have employed multiple Piezoelectric Harvester in parallel for achieving high output power. Further evaluation is carried out in two stage; at first we plot graph, later further evaluation is carried out with several existing methodology and it is observed that our model achieves massive output power.
\end{abstract}

Keywords: Energy Harvesting, MM-PZT, Acoustic harvester.

\section{INTRODUCTION}

Recent years have seen a several global problem such as environment pollution, global warming, energy crisis and other human responsible problem and this is increasing in exponential way; energy is one of the important phenomena, which is focused in this research. Moreover, there are different types of sources to get clean and renewable energy such as bio-energy, kinetic energy and solar energy these energy have the capabilities to replace the traditional energy sources such as fossil fuels. Furthermore, these energy are environmental friendly as well as more sustainable. Considering the vibrant and wide application of these energy research an industry folks have given fair amount of effort to energy harvesting technologies; energy harvesting technologies mainly focuses on scavenging waste energy available in the environment such as water, wind, radiation, light and vibration are converted into the electrical energy;

Revised Manuscript Received on April 18, 2020.

* Correspondence Author

M. Sreenivasulu*, Research Scholar, ECE Dept, JNTUH, Hyderabad \& Assoc. Professor, Samskruti College of Engg. \& Tech,Hyderabad501301. E-mail: srinuvas42@gmail.com

Dr. V. Usha Shree, Principal \& Professor, Dept. of ECE, JRBEC, Affiliated to JNTUH, Hyderabad, India. E-Mail: valasani_usha1@yahoo.com

Dr. P. Chandrasekhar Reddy, Professor in ECE, BOS Chairman, Dept. of ECE, JNTUCE, JNTUH, Hyderabad. E-Mail: drpcsreddy@gmail.com

(C) The Authors. Published by Blue Eyes Intelligence Engineering and Sciences Publication (BEIESP). This is an open access article under the CC BY-NC-ND license (http://creativecommons.org/licenses/by-nc-nd/4.0/) portable devices as sensor nodes for Internet of Things, transport tracking and health monitoring; Energy harvesting these are designed for low power devices[1]. Vibrational energy harvesting is considered one of the solution for technique i.e. conversion of kinetic energy to electrical energy is carried out by electromagnetic transduction mechanism, electrostatic and piezoelectric; among these piezoelectric have been considered by researcher due to its higher rate of conversion of mechanical energy into electrical energy and hence it provides the higher output power and power density. In addition; PEH does not require any kind of external electrical input, In general $\mathrm{PEH}$ transforms vibrational energy to mechanical strain through piezoelectric effect, later this effect creates voltage around the piezoelectric layer; further it is very simple and has ease of fabrication; this results in creating the higher strain through integrating of proof mass at cantilever. However the main issue is in its volumetric size as it is getting reduced day by day. Mechanical energy harvesting has shown a promising technology since it uses piezoelectric components; this transfers the vibration energy into electrical energy and further this electrical energy can be stored and regulated. This also might replace the batteries in various devices such as pressure sensor system, implementable medical electronic and micro sensor networks. Vibrational energy harvesting is considered one of the solution for portable devices as sensor nodes for Internet of Things, transport tracking and health monitoring. The below figure shows the energy harvesting based on piezoelectric devices; it comprises five components. First component is transducer, which helps in converting vibration energy into electrical energy; transducer is a sensor, which is modeled through current source parallel with resistor and capacitor. Moreover, electrical energy generated as an output for transducer is irregular and strong; hence DC supply source is implied and later these energy are stored as supper capacitor or battery. Furthermore, before using energy voltage regulated is required for regulating the suitable voltage [3].

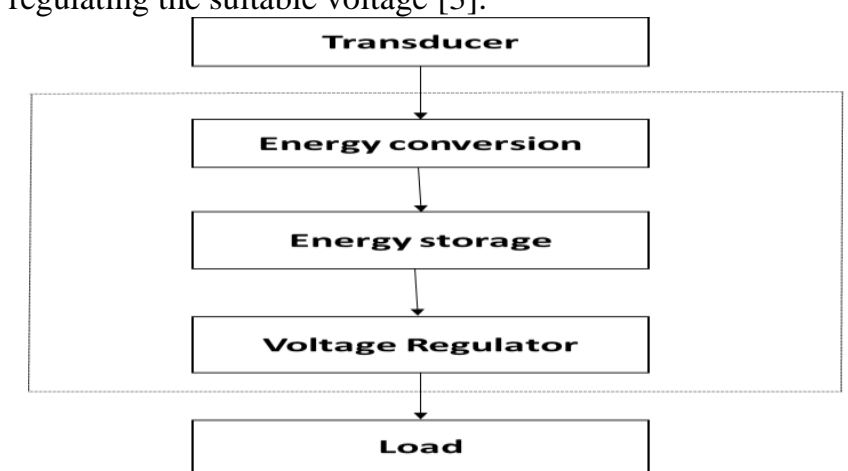

Figure 1: energy harvesting

Published By:

Blue Eyes Intelligence Engineering

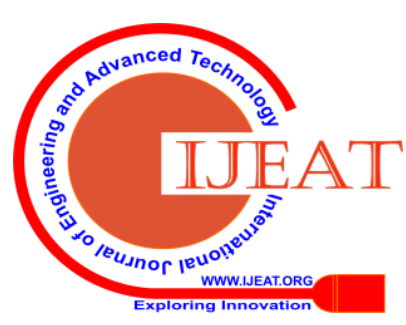


Most popular piezoelectric material is PZT (zirconate titnate), PVDF, AIN, ZNO; all these material have advantage and disadvantage considering the specific application; for instance quality check of water in river requires flexible substrate whereas for peacemaker lead free harvester is required. Moreover, AIN is one of the material that are compatible with technologies of micro-fabrication [4] [5].

\subsection{Motivation and contribution of research work}

Throughout the research, it is observed that many researcher have focused on cantilever tuning through varying stiffness. In this research work, we consider multiple PZT for achieving the high output power; further contribution of this research work is listed below.

1. We propose a mechanism for acoustic energy harvesting named MM-PEH (Multilayered multiple Piezoelectric Harvester).

2. Proposed mechanism has multiple multilayer-PEH model where many multilayered -PEH is placed parallely; In general multilayer-PEH comprises six layered which is placed one over the other with their properties.

3. The main aim of MM-PEH (Multilayered multiple Piezoelectric Harvester) mechanism is to achieve higher output power with low volume.

4. MM-PEH (Multilayered multiple Piezoelectric Harvester) is evaluated considering the through comparative analysis.

This research work is organized like any standard research work, here in first section we discuss the background of energy harvesting and its need later in the same section we discuss the energy harvesting method and the material along with its importance. Further sub-section discuss the motivation and contribution of this particular research work. Second section focus on discussing existing mechanism along with its shortcomings; third section presents MM$\mathrm{PEH}$ mechanism along with mathematical notation. MM$\mathrm{PEH}$ is evaluated in fourth section through graphical representation and comparative analysis is carried out to prove the efficiency of the model.

\section{LITERATURE SURVEY}

[6] Presented a particular mode named d33 MEMS for harvesting energy which comprises silicon substrate and PMN-PT thick film; further d33 coupling was exploited through interdigitated electrodes. Moreover [7] used a fabrication method for thinning a bulk PMN-PT; thus developed fabricated prototype has an active volume of 0.418 observes output power of 7.182; later after few years same group implied PZT thick film on substrate beryllium bronzed with the help of bonding and thinning technique for fabricating PZT MEMS [8]; fabricated device with active volume of $30.6 \mathrm{~mm} 3$ observes output of 0.979 with acceleration of $3.5 \mathrm{~g}$ considering frequency as $77.2 \mathrm{~Hz}$. [9]Presented a model where PZT layers were deposited on both sides of stainless steel; this is used through customization for fabricating energy device; further prototype developed with dimension of 9mm X 6 MM X 90 $\mu \mathrm{m}$ is evaluated under the base excitation of $140.8 \mathrm{~Hz}$ at $1.5 \mathrm{~g}$ observes output power of $413 \mu \mathrm{W}$. [10] Presented a mems cantilever that was based on silicon and uses aluminum nitride for piezoelectric layer; further it is embedded into the peacemaker with $6 \mathrm{~mm}$ diameter and 40 mm length. Moreover, harvester observes power output density of 454 and 97 considering heart rate of $240 \mathrm{bpm}$ and 60 bpm respectively. [11] Proposed pressure sensing device and self-powered viscosity for given application in the microfluidic model. Here the device comprises PVDF layer, which is deposited on PDMS substrate for forming microchip; further the device was evaluated under water droplet found in microfluidic model. The proposed voltage signals are generated through the harvester is analyzed for measuring the viscosity and pressure of microfluid. In [12] low frequency MEMS were proposed for harvesting energy which comprises PZT thin film along with proof mass on flexible substrate of phosphor bronze; Here a rectangular hole is created on the beam harvester for minimizing resonance frequency. Further power output of 1713.58 and $216.66 \mathrm{mWw}$ with acceleration of $34.3 \mathrm{~Hz}$ at $1.5 \mathrm{~g}$ for load resistance $60 \mathrm{k} \Omega$. [13] Used $\mathrm{LaNiO} 3$ along with thin film is deposited on given ultra-thin metallic foil of Ni cr. Here metal foil gives the compressive stress in PZT and compared to the substrate of silicon; further thermal expansion is utilized for imposing large biaxial stresses and also makes stress tuned in fabrication method. Moreover prototype developed with $2 \mathrm{~mm} \mathrm{X} \mathrm{4mm} \mathrm{X} 25 \mu \mathrm{m}$ for punching process. It is observed that this prototype observes output power of $5.6 \mu \mathrm{W}$ in given $11 \mathrm{k} \Omega$ load resistance.

\section{PROPOSED METHODOLOGY}

\subsection{Preliminaries}

The below equation presents the dynamic motion PZT, here an indicates any deflection in spring, $\mathbb{Z}$ is displacement of input, $\mathbb{D}_{\mathbb{f}}$ indicates damping coefficient (electrical) and $\mathbb{D}_{\mathrm{m}}$ also indicates damping coefficient(mechanical) .

$$
-\mathrm{m}_{\mathrm{Z}} \ddot{\mathrm{z}}=1 \mathrm{l} \mathrm{a}+\left(\mathbb{D}_{\mathbb{f}}+D_{\mathrm{m}}\right) \dot{\mathrm{a}}+\text { mä }
$$

Moreover there occurs a phenomena where the energy conservation is maximum, this phenomena occurs when both vibration frequency and resonant frequency matches, else the efficiency remain on lower side. However, the model has high $\mathrm{Q}$, which results in small operating bandwidth and it is not ideal to variable frequency vibrations. Furthermore, PZT possesses multiple DOF (Degree of Freedom) and the efficiency of the model depends on the proof mass $m$ and stiffness $k$; hence, several researcher focused on designing the resonant frequencies generator to enhance the maximum o/p power. However, it is possible to design such energy harvester where the resonant frequency can be matched to the vibrant frequency; further through the review analysis it is observed that there is a demand of energy harvester which is capable of operating at the wider bandwidth.

1.3 MM-PEH (Multilayered multiple Piezoelectric Harvester )

1.3.1 Electrical equivalency of MM-PEH (Multilayered multiple Piezoelectric Harvester)
Published By:

\& Sciences Publication (c) Copyright: All rights reserved.

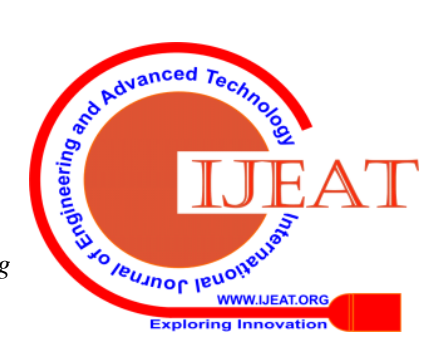




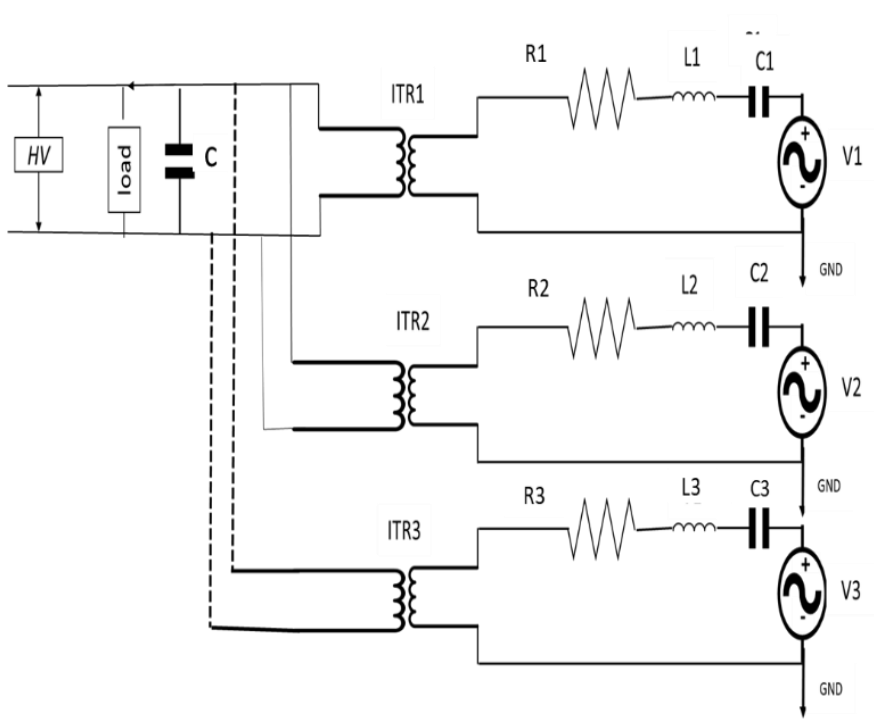

Figure 2 elctrical representation of MM-PEH

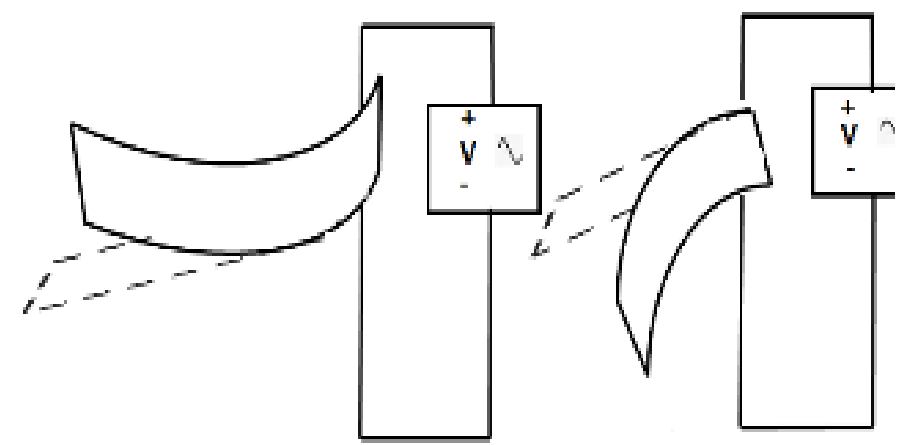

Figure 3 effect of Piezoelectronic

In this section, we focus on designing the multiple PZT, in the previous research work we have focused on developing the single PZT for energy harvesting with multiple layer; further extending the previous research work we extend our work and add multiple multilayered PEH for achieving high output power. At first we start with two cantilever beams in parallel, considering the vertical vibration for both of them. The above diagram i.e. figure 2 presents the equivalent circuit of MM-PEH model and comprises various parameter voltage source indicates as $V_{1}, V_{2}$ and $V_{3} ; C_{1}, C_{2}$ and $C_{3}$ indicates capacitors; $L_{1}, L_{2}$ and $L_{3}$ are Inductors. HV indicates the Harmonic voltage; figure 3 presents effect of Piezoelectric. In this mechanism, several cantilevers are attached to the main beam with their position with test mass . further $\mathbb{M}$ indicates the beam length, with this parameter we compute the bending and torsion which is depicted in the below equation.

$$
\begin{gathered}
\mathbb{z}(\mathrm{y}, \mathrm{u})=\mathrm{r}_{0}(\mathrm{un}) \Phi_{0}(\mathrm{y})+\mathrm{r}_{1}(\mathrm{un}) \Phi_{1}(\mathrm{y}) \\
+\mathrm{r}_{2}(\mathrm{un}) \Phi_{2}(\mathrm{y}) \ldots
\end{gathered}
$$

$$
\begin{gathered}
\Phi_{i}(\mathbb{y})=\cosh \frac{\Lambda_{j} \mathbb{Y}}{\mathbb{M}}-\cos \frac{\Lambda_{i} \mathbb{Y}}{\mathbb{M}}-\frac{\cosh \Lambda_{i}-\cos \Lambda_{i}}{\sinh \Lambda_{j}-\sin \Lambda_{j}} \\
\mathbb{y}\left(\sinh \frac{\Lambda_{j} \mathbb{Y}}{\mathbb{M}}-\sin \frac{\Lambda_{j} \mathbb{y}}{\mathbb{M}}\right)
\end{gathered}
$$

$$
\Psi_{j}(\mathrm{y})=\sin \frac{\mathrm{j} \pi \mathrm{y}}{\mathbb{M}} .
$$

Further, we compute the deformation energy or DF and depicted in equation 6 ; in here $\mathbb{F}$ indicates the mechanical property, $G J$ indicates section torsion constant; it is product of modulus of rigidity and section torsion constant. $E I$ is bending force couple, which is product of MOI (Moment of Inertia). The below diagram shows MM-PEH in figure 4 where multiple cantilever beam are placed parallely; this structure comprises beam where beams are accelerated equally. Here beam supports the multiple small cantilevers.M1to M6 indicates the proof mass.

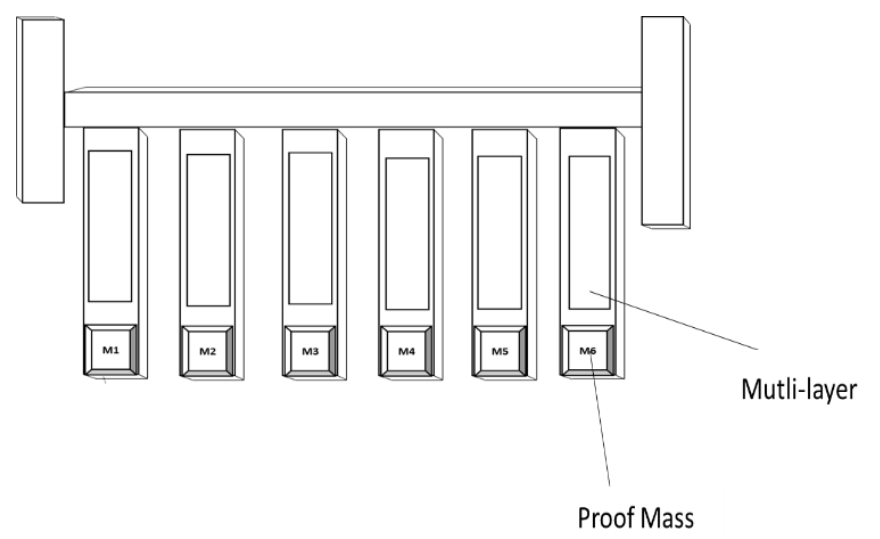

Figure 4 MM_PEH

$$
\begin{aligned}
& \mathbb{V}(\text { खा })=\frac{1}{2} \int_{0}^{\mathbb{M}} E I y^{\prime \prime 2}(\mathbb{y}, \text {, }) d \mathbb{y} \\
& +\frac{1}{2} \int_{0}^{\mathbb{M}} G J \theta^{\prime \prime 2}(\mathrm{y}, \text { un }) d x \\
& +\frac{1}{2} \sum_{j=1}^{2} \mathbb{1}_{k} \mathbb{Z}_{k}^{2}
\end{aligned}
$$

The above equation constitutes three distinctive term, first term represents the deformation energy, second term is torsional deformation energy whereas third term indicates the cantilever deformation energy. Further, we compute the KE (Kinetic Energy) of the model and formulated through the given equation.

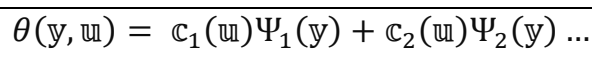

In the above equation 2 and $3, \phi$ and $\psi$ both are shape function, $\mathbb{r}_{j}\left(\right.$ (n) and $\mathbb{C}_{2}(\mathbb{m})$ are considered as the coordinates. Once torsion and bending are calculated we compute shape of node through 4 and 5.

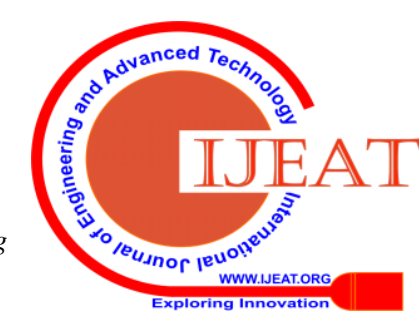




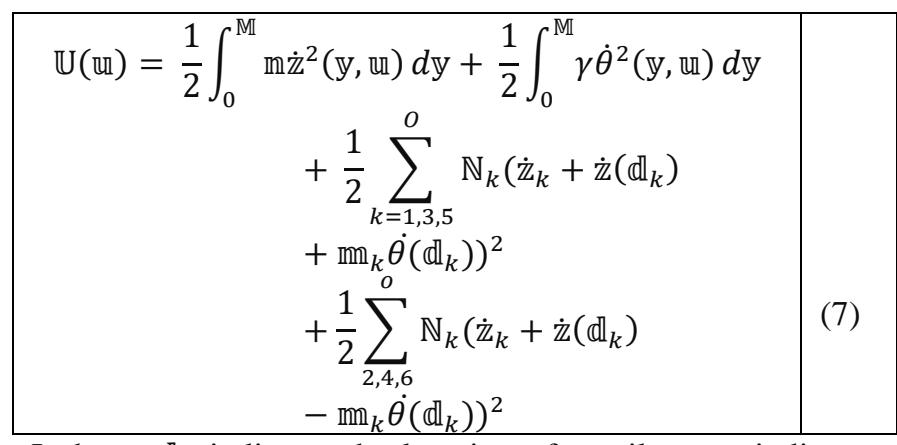

In here, $\mathbb{d}_{k}$ indicates the location of cantilever $n$ indicates the total cantilevers and $m$ indicates mass per unit length. In above equation i.e. equation 7 there are four KE (Kinetic Energy) term, first term, second term and third term indicates the kinetic energy of bending, beam torsion, odd number cantilever and even number cantilever. Further substituting the equation 8 and equation 9 into 6 we formulate equation 10 .

$$
\mathbb{Z}^{\prime \prime}(\mathbb{y}, \text { 니 })=\mathbb{r}_{0} \Phi_{0}^{\prime \prime}(\mathbb{y})+\mathbb{r}_{1} \Phi_{1}^{\prime \prime}(x)+\mathbb{r}_{2} \Phi_{2}^{\prime \prime}(x)
$$

$$
\theta^{\prime}(\mathbb{y}, \text { ㄸ })=\mathbb{c}_{1} \Psi_{1}^{\prime}(\mathbb{y})+\mathbb{c}_{2} \psi_{2}^{\prime}(\mathbb{y})
$$

Hence the system deformation energy can be derived as:

$\mathbb{V}$
$=\frac{1}{2} \int_{0}^{\mathbb{M}} E I\left(\mathbb{r}_{0} \Phi_{0}^{\prime \prime}(\mathbb{y})+\mathbb{r}_{1} \Phi_{1}^{\prime \prime}(\mathbb{y})\right.$
$\left.+\mathbb{r}_{2} \Phi_{2}^{\prime \prime}(\mathbb{y})\right)^{2} d x+\frac{1}{2} \int_{0}^{L} G J\left(\mathbb{c}_{1} \Psi_{1}^{\prime}(\mathbb{y})\right.$
$\left.+\mathbb{c}_{2} \Psi_{2}^{\prime}(\mathbb{y})\right)^{2} d \mathbb{y}+\frac{1}{2} \sum_{j=1}^{2} \mathbb{1}_{k} \mathbb{Z}_{k}^{2}$

Furthermore substituting $\dot{\mathbb{Z}}((\mathbb{y}$, un $))$ and $\theta^{\prime}((\mathbb{y}$, un $))$ of the equation 11 and 12, the kinetic energy can be formulated as given in equation 12 .

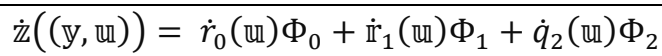

$$
\theta^{\prime}(\mathrm{y}, \text { 凹n })=\mathbb{C}_{1} \Psi_{1}^{\prime}+\mathbb{C}_{2} \Psi_{2}^{\prime}
$$

$$
\begin{aligned}
& \mathbb{V} \\
& =\frac{1}{2} \int_{0}^{\mathbb{M}} E I\left(\mathbb{r}_{0} \Phi_{0}^{\prime \prime}(\mathrm{y})+\mathbb{r}_{1} \Phi_{1}^{\prime \prime}(\mathbb{y})\right. \\
& \left.+\mathbb{r}_{2} \Phi_{2}^{\prime \prime}(\mathbb{y})\right)^{2} d \mathrm{y}+\frac{1}{2} \int_{0}^{\mathbb{M}} G J\left(\mathbb{C}_{1} \Psi_{1}^{\prime}(\mathrm{y})\right. \\
& \left.+\mathbb{C}_{2} \Psi_{2}^{\prime}(\mathbb{y})\right)^{2} d x+\frac{1}{2} \sum_{k=1}^{2} \mathbb{1}_{k} \mathbb{Z}_{k}^{2} .
\end{aligned}
$$

\begin{tabular}{|c|c|}
\hline$\dot{\theta}(\mathrm{y}, \mathrm{u})=\dot{\mathbb{C}}_{1}($ u $) \psi_{1}^{\prime}+\dot{\mathbb{C}}_{2}($ u $) \psi_{2}^{\prime}$ & $(15)$ \\
\hline 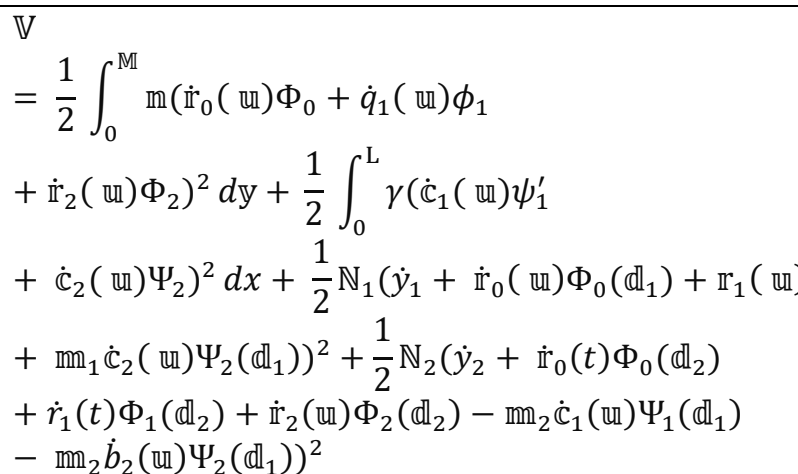 & $\begin{array}{l}(1 \\
6)\end{array}$ \\
\hline
\end{tabular}

Moreover, in order to compute the motion through deformation energy and kinetic energy we make use of Lagrange equation.

$\dot{\mathbb{Z}}(\mathrm{y}, u)=\dot{\mathrm{r}}_{0}\left(\right.$ u) $\phi_{0}+\dot{\mathrm{r}}_{1}\left(\right.$ m) $\phi_{1}+\dot{\mathrm{r}}_{2}($ m) $) \Phi_{2}$
Lagrange equation can be written as:

\begin{tabular}{|c|c|}
$\mathbb{R}_{k}=\frac{\mathrm{d}}{\mathrm{d} \mathbb{}}\left(\frac{\partial \mathbb{U}}{\partial \dot{\mathbb{r}}_{k}}\right)-\frac{\partial \mathbb{U}}{\partial \mathbb{r}_{k}}+\frac{\partial \xi}{\partial \dot{\mathbb{r}}_{k}}+\frac{\partial \mathbb{V}}{\partial \dot{\mathbb{r}}_{k}}$ & (17) \\
$=\frac{\partial(\delta \mathbb{X})}{\partial(\delta k)} \quad$ for $k=1,2, \ldots, \mathbb{O}$ & \\
\hline
\end{tabular} Moreover this equation is used for generalized coordinate $\mathbb{r}_{k}$ where $\xi$ indicates the damper contribution, $\delta \mathbb{r}_{k}$ indicates the $\mathrm{VD}$ (Virtual displacement) and $\delta \mathbb{X i n d i c a t e s}$ the incremental work .

Further lets assume that in system there is occurrence of under free vibration, this results that incremental work is null and hence other terms need to derived; once we determine all the term in equation 15 , the equation of motion is formulated through equation 18.

$$
\widetilde{\mathbb{N}} \times\left[\begin{array}{c}
\ddot{\mathbb{r}}_{0} \\
\ddot{\mathbb{r}}_{1} \\
\ddot{\mathbb{r}}_{2} \\
\ddot{\mathbb{C}}_{1} \\
\ddot{\mathbb{C}}_{2} \\
\ddot{y}_{1} \\
\ddot{y}_{2}
\end{array}\right]+\widetilde{\mathbb{D}} \times\left[\begin{array}{c}
\dot{\mathbb{r}}_{0} \\
\dot{\mathbb{r}}_{1} \\
\dot{\mathbb{r}}_{2} \\
\dot{\mathbb{C}}_{1} \\
\dot{\mathbb{C}}_{2} \\
\dot{y}_{1} \\
\dot{y}_{2}
\end{array}\right]+\widetilde{\mathbb{I}} \times\left[\begin{array}{c}
\mathbb{r}_{0} \\
\mathbb{r}_{1} \\
\mathbb{r}_{2} \\
\mathbb{C}_{1} \\
\mathbb{C}_{2} \\
y_{1} \\
y_{2}
\end{array}\right]=0
$$

Above equation is in the form of matrix, here $\widetilde{\mathbb{D}}, \widetilde{\mathbb{\mathbb { I }}}$ and $\widetilde{\mathbb{N}}$ are matrices which indicates effective mass, $\widetilde{\mathbb{D}}$ is damping.; consider damping as null row and column can be known.; $\mathbb{r}_{0}-\mathbb{r}_{2}$ and $\mathbb{r}_{0}-\mathbb{r}_{2}$ are the deformation energy and kinetic energy.

Moreover, through the above function and formula we can predict the transmission of multiple cantilever model; this can be achieved through using the mass matrices and stiffness metrics. Moreover, the above analysis can be applied to the multiple cantilever. In order to apply for multiple cantilever mass and stiffness are expanded. The above equation can be formulated as equation 19 ,

Published By:

Blue Eyes Intelligence Engineering

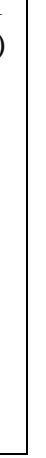

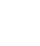




$$
\left[\begin{array}{ll}
\widetilde{\mathbb{N}}_{00} & \widetilde{\mathbb{N}}_{01} \\
\widetilde{\mathbb{N}}_{10} & \widetilde{\mathbb{N}}_{11}
\end{array}\right] \times\left[\begin{array}{l}
\ddot{\mathbb{N}}_{0} \\
\ddot{\mathbb{N}}_{1} \\
\ddot{\mathbb{N}}_{2} \\
\ddot{\mathbb{C}}_{1} \\
\ddot{\mathbb{C}}_{2} \\
\ddot{\mathbb{Z}}_{1} \\
\ddot{\mathbb{Z}}_{2}
\end{array}\right]+\left[\begin{array}{cc}
0 & 0 \\
0 & \widetilde{\mathbb{I}}_{11}
\end{array}\right] \times\left[\begin{array}{l}
\mathbb{r}_{0} \\
\mathbb{r}_{1} \\
\mathbb{r}_{2} \\
\mathbb{C}_{1} \\
\mathbb{C}_{2} \\
\mathbb{Z}_{1} \\
\mathbb{Z}_{2}
\end{array}\right]=0
$$

In the above equation, $\widetilde{\mathbb{N}}_{00}$ is FRFC (First Row First column) of the matrix $\widetilde{\mathbb{N}}$ i.e. $\widetilde{\mathbb{N}}_{11}$ and $\widetilde{\mathbb{N}}_{10}$ are 6 x6 and 1 X6 matrix; similarly $\widetilde{\mathbb{N}}_{01}$ is $1 \mathrm{X} 6$ matrix, $\ddot{\mathbb{r}}_{0}$ is excitation. Through the equation we observe that there does not exist any terms in matrix in $\widetilde{\mathbb{1}}$, this causes the FRFC to be zero. Hence we formulate the equation 18 as below in equation 20 and equation 21 . Furthermore as we observe $\ddot{\mathbb{r}}_{0}$ is excitation, a force equation is developed through equation 20 which generates $\ddot{\underline{x}}_{0}$ and equation 21 computes response occurred from excitation.

\begin{tabular}{|l|l|}
\hline $\mathbb{N}_{00} \mathbb{r}_{0}+\mathbb{N}_{01} \ddot{\underline{x}}_{00}=\mathbb{G}$ & (20) \\
\hline
\end{tabular}

\begin{tabular}{|l|l|}
\hline $\mathbb{N}_{00} \ddot{\mathbb{Y}}_{0}+\mathbb{N}_{11} \ddot{\ddot{\mathbb{x}}}_{0}+K_{11} \underline{\mathbb{T}}_{00}$ & [21] \\
\hline
\end{tabular}

In the above equation, input force on the model is denoted as $\mathbb{G}$; response vector and $\underline{\mathbb{r}}_{00}$ both are same, hence we assume harmonic response for $\underline{\ddot{\mathbb{x}}}_{0}$ and $\underline{\mathbb{r}}_{00}$ as given equation 21 and 22.

$$
\ddot{\underline{r}}_{0}=\ddot{\mathbb{B}}_{0} \mathbb{f}^{\mathrm{i} \omega \text { un }}
$$

$$
\underline{\mathbb{r}}_{00}=\underline{\mathbb{B}}_{00} \mathbb{f}^{\mathrm{i} \omega \mathbb{u}}
$$

Furthermore, these equation are substituted in 21 and 22; equation can be restructured and written through 23 and 24

$$
\underline{\mathbb{B}}_{00}=\left(-1 /\left[\mathbb{1}_{11}-\Omega^{2} \mathbb{N}_{11}\right]^{-1}\right) \mathbb{N}_{10} \ddot{\mathbb{B}}_{0}
$$

$$
\ddot{\mathbb{B}}_{00}=\left(1 / \Omega^{2}\left[\mathbb{1}_{11}-\Omega^{2} \mathbb{N}_{11}\right]\right) \mathbb{N}_{10} \ddot{A}_{0}
$$

Now, the model output needs to be computed and this is formulated through the equation 24; where beam location is denoted through $\mathbb{e}_{k}$

$$
\begin{aligned}
\ddot{\mathbb{X}}\left(\mathbb{e}_{k}\right)=\ddot{\mathbb{r}}_{0} \Phi_{0}\left(\mathbb{e}_{k}\right)+\ddot{\mathbb{r}}_{1} \Phi_{1}\left(\mathbb{e}_{k}\right)+\ddot{\mathbb{r}}_{2} \Phi_{2}\left(\mathbb{e}_{k}\right) \\
+\mathbb{C}_{1} \Psi_{1}\left(\mathbb{e}_{k}\right) \\
+\mathbb{C}_{2} \Psi_{2}\left(\mathbb{e}_{k}\right) \ldots \text { for } k=1,2, \ldots
\end{aligned}
$$

The model output equation can parted into several distinctive terms; first term denotes the input excitation, second term indicates bending response and third term indicates second mode shape, fourth term indicates the torsion response. Further model output is given as:

$$
\ddot{\mathbb{X}}=\left[\begin{array}{l}
\Phi_{0}\left(\mathbb{e}_{1}\right) \\
\Phi_{0}\left(\mathbb{e}_{2}\right)
\end{array}\right] \cdot \ddot{\mathbb{B}}_{0}+\tilde{\phi} \cdot \underline{\mathbb{B}}_{00}
$$

In the above equation we use excitation matrix that can be denoted as $\left[\begin{array}{l}\Phi_{0}\left(\mathbb{e}_{1}\right) \\ \Phi_{0}\left(\mathbb{e}_{2}\right)\end{array}\right]$; we recreate the equation 23 and compute the output response through below equation.

$$
\underline{\ddot{X}}=\ddot{\mathbb{B}}_{0}\left\{\Omega^{2} \widetilde{\Phi}\left[\mathbb{1}_{11}-\omega^{2} \mathbb{N}_{11}\right]^{-1} \mathbb{N}_{10}+\left[\begin{array}{l}
\Phi_{0}\left(\mathbb{e}_{1}\right) \\
\Phi_{0}\left(\mathbb{e}_{2}\right)
\end{array}\right]\right\}
$$

Further, multiple cantilever transmissibility is computed through the below equation.

$$
\begin{aligned}
\underline{\mathbb{U}}=\frac{\ddot{\ddot{X}}}{\ddot{\mathbb{B}}_{0}}=\frac{\text { Output }}{\text { Input }} & \\
= & \Omega^{2} \widetilde{\Phi}\left[\mathbb{1}_{11}\right. \\
& \left.-\omega^{2} \mathbb{N}_{11}\right]^{-1} \cdot \mathbb{N}_{10}+\left[\begin{array}{l}
\Phi_{0}\left(\mathbb{e}_{1}\right) \\
\Phi_{0}\left(\mathbb{e}_{2}\right)
\end{array}\right]
\end{aligned}
$$

Hence, through the above process we successfully design the MM-PEH prototype for harvesting energy.

\section{PERFORMANCE EVALUATION}

In this section we evaluate the proposed MMPEH(Multilayered multiple Piezoelectric Harvester ); for evaluation system configuration includes i7 processor with 2GB Nvidia Graphics loaded with $8 \mathrm{~GB}$, python is used as a programming language. Moreover, evaluation is carried out in two sub-section; first sub-section presents the graphical evaluation considering the parameter as displacement, current and power with varying frequency and identifying the peak value of same. Second sub-section presents the comparative analysis with various state-of-art technique and existing mechanism for harvesting energy.

\subsection{Graphical Evaluation}

\subsubsection{Displacement vs Frequency}

In general Displacement is defined as the action of change in particular object; in here displacement is the distance among the given peaks of vibration; it is considered to be one of the important metrics for evaluation of any harvesting device. Frequency is the total number of times that repetitive motion in particular time; in here through the graph we observe the displacement at different frequency as depicted in the figure 5. Moreover, peak value is observed at frequency of 139.5 with displacement 74.84 .

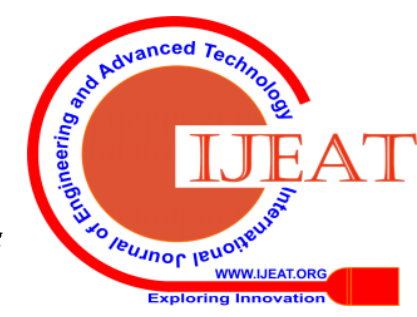




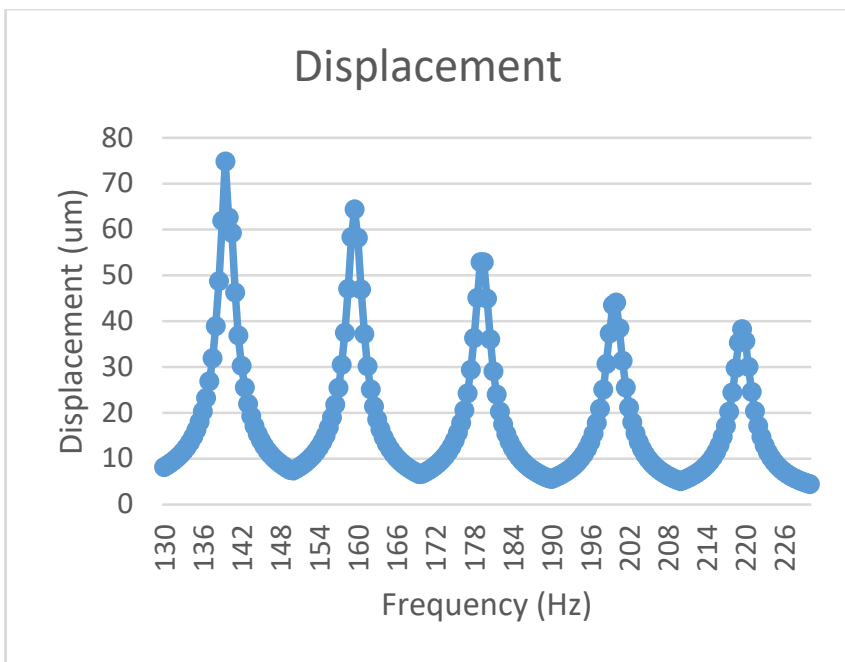

\subsubsection{Current}

Figure 5 Displacement vs Frequency

In below figure i.e. figure 6 , graph is plotted as current vs frequency; here current value is observed by varying the frequency and find the peak current. In here peak current value is observed at 159.5 with current value as 390017.

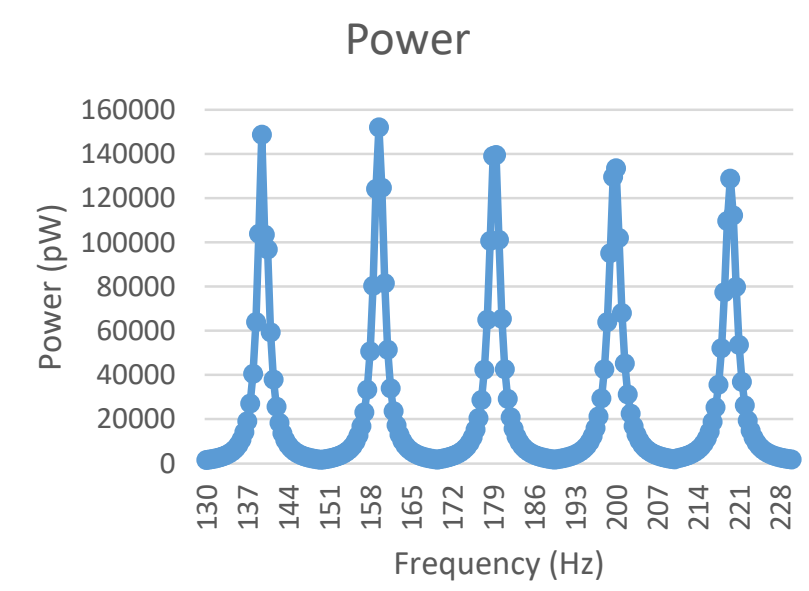

\subsection{Comparative analysis}

In this section, we evaluate our proposed mechanism through comparing with various methodology considering different metrics given in table 3 . In table 3 first column shows the different mechanism for energy harvesting, second column presents the technology adopted, third column presents volume of material used, fourth column presents acceleration, fifth column gives frequency range, sixth column presents column presents the NPD, seventh column presents Volume and last column presents the

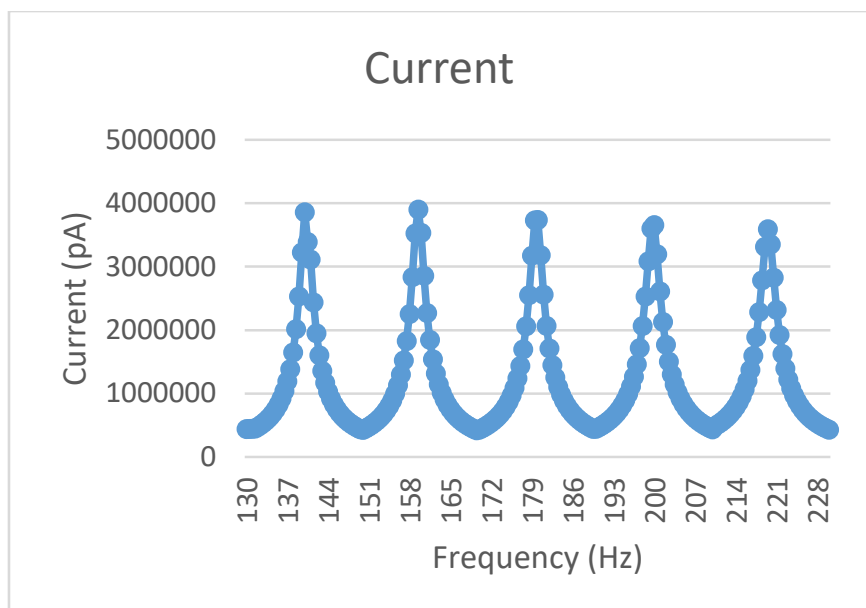

Figure 6Current Vs Frequency

\subsubsection{Power}

Power plays an important role in harvesting energy; as similar to above figure i.e. figure 5 and figure 6 , we analyze the variation of power at different frequency and find the peak value. In below figure peak value of power is observed at frequency of 159.5 with value of 152124.5 and it is computed in PW. Power. Further we evaluate proposed model through comparing with these state-of-art technique along with existing model. Furthermore, in case of existing model acceleration is 0.5 which is below average and our proposed model achieves massive acceleration of $9.8 \mathrm{~m} \mathrm{~m} / \mathrm{s} 2$; the higher acceleration indicates that better efficiency of model. Further, considering other parameter such as frequency range proposed model has frequency range of 130-230 in comparison with existing model of 227-286. Further output power is main focus of this research work and through the table we observe that our proposed model achieves massive output of $106.15 \square \mathrm{W}$ in comparison with output power of $0.61 \square \square \mathrm{W}$.

Normalized power density is one of the performance metrics; NPD is computed as the ratio among harvested power and harvester size volume; considering this metric NPD comparison is carried out between the several state-ofart technique and proposed prototype. Furthermore through table 3 we observe that [16] possesses good amount of NPD; here PZT material was used as an active layer; [17] also observes fair NPD considering steel as substrate, however this cannot be considered as energy harvester since it possesses large dimensions. Existing mechanism achieves NPD of 595.12 $\square \mathrm{W}$ whereas our model achieves NPD of massive 1757.

Table 1 comparison of various methodologies considerign different parameter

\begin{tabular}{|c|c|c|c|c|c|c|c|}
\hline Ref. & Te-ch. & Sub- strate & $\begin{array}{c}\text { Accel. } \\
{[\mathrm{m} / \mathrm{s} 2]}\end{array}$ & Freq. Range [Hz] & $\begin{array}{c}\text { NPD } \\
{\left[\begin{array}{l}\text { W.cm- } \\
\text { 3m-2s4] }\end{array}\right.}\end{array}$ & $\begin{array}{c}\text { Volume } \\
{[\mathrm{mm} 3]}\end{array}$ \\
\hline$[14]$ & $\mathrm{Pi}$ & $\mathrm{Si}$ & 9.8 & $30-47$ & 0.52 & 10 & 0.5 \\
\hline$[15]$ & $\mathrm{Pi}$ & $\mathrm{Si}$ & 1.96 & $71.8-188.4$ & 1.97 & 18 & 0.136 \\
\hline$[16]$ & $\mathrm{Pi}$ & $\mathrm{Si}$ & $\sim 9.8$ & $500-1350$ & \#\#\#\#\#\# & 0.021 & 2000 \\
\hline
\end{tabular}

Published By:

Blue Eyes Intelligence Engineering
\& Sciences Intelligence

(C) Copyright: All rights reserved.

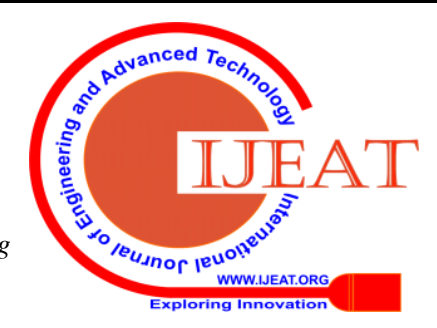




\begin{tabular}{|c|c|c|c|c|c|c|c|}
\hline [17] & $\mathrm{Pi}$ & Steel & 0.392 & 04-Aug & \#\#\#\#\#\# & 1419.35 & 2610 \\
\hline [18] & $\mathrm{El}^{*}$ & $\mathrm{Si}$ & 1.372 & $520-591$ & 1.63 & 27.1 & 0.083 \\
\hline [19] & $\mathrm{El}$ & $\mathrm{Si}$ & 0.9 & $148-172$ & 32.68 & 187 & 4.95 \\
\hline [20] & $\mathrm{El}$ & $\mathrm{Si}$ & 12.8 & 590-641 & 0.31 & 295.8 & 14.8 \\
\hline [21] & Eg & $\mathrm{Si}$ & 9.8 & $840-1490$ & \#\#\#\#\#\# & 35 & 0.0055 \\
\hline [22] & $\mathrm{Eg}$ & FR4 & 10 & 190-244 & 0.0045 & 43.35 & 0.45 \\
\hline [23] & $\mathrm{Pi}$ & $\mathrm{Si}$ & 19.6 & $\begin{array}{c}859.9- \\
924.5\end{array}$ & 1.91 & 112 & 82.24 \\
\hline [24] & $\mathrm{Pi}$ & $\mathrm{Si}$ & 0.6 & 210 & 990 & 5 & 1.78 \\
\hline [25] & $\mathrm{Pi}$ & $\mathrm{Si}$ & 0.5 & 449.3 & 6400 & 112 & 180 \\
\hline [26] & $\mathrm{Pi}$ & $\mathrm{Al}$ & 0.98 & $\begin{array}{c}35.4- \\
126.4\end{array}$ & 0.022 & 52020 & 1.1 \\
\hline [27] & $\mathrm{Pi}$ & $\mathrm{Si}$ & 0.5 & $387-398$ & 32.55 & 6500 & 52.9 \\
\hline$[28]$ & $\mathrm{Pi}$ & $\mathrm{Si}$ & 7.84 & Dec-26 & 0.008 & 175 & 087 \\
\hline ES[29] & $\overline{\mathrm{Pi}}$ & $\overline{\mathrm{Si}}$ & 0.5 & $227-286$ & 595.12 & 4.1 & 0.61 \\
\hline $\begin{array}{l}\text { PS(MM- } \\
\text { PEH) }\end{array}$ & $\mathbf{P i}$ & Si & 9.8 & $130-230$ & 1757 & 0.62906 & 106.15 \\
\hline
\end{tabular}

V. CONCLUSION

In this research work we propose a novel mechanism named MM-PEH mechanism for energy harvesting; the main aim of this mechanism is to obtain maximum output power with low volumetric size of material. Further this is achieved through placing multiple multilayered PZT material parallely. Moreover MM-PEH is evaluated in two section; at first we plot a graph of displacement, power and current by varying the frequency to find the peak value of each; later comparative analysis is carried out considering the several important metrics with fifteen state-of-art technique along with existing methodology. Furthermore, through evaluation it is observed that volumetric size of our prototype is very much less i.e. $3.47 \mathrm{~mm} 3$ lesser than the existing protocol. In terms of power our model achieves $105.54 \square \mathrm{W}$ more output than existing protocol; similarly in terms of normalized power density proposed model achieves $1161.8 \square$ W.cm more than the existing model. Hence, through the analysis it is observed that this could be first potential work that has achieved such high output power with less volume; however in future for real time scenario this model needs to be evaluated considering the other parameters and it can be further optimized.

\section{REFERENCE}

1. S. Guruacharya and E. Hossain, "Self-Sustainability of Energy Harvesting Systems: Concept, Analysis, and Design," in IEEE Transactions on Green Communications and Networking, vol. 2, no. 1, pp. 175-192, March 2018.

2. H. Song et al., "Ultra-Low Resonant Piezoelectric MEMS Energy Harvester With High Power Density," in Journal of Microelectromechanical Systems, vol. 26, no. 6, pp. 1226-1234, Dec. 2017.

3. T. Ruan, Z. J. Chew and M. Zhu, "Energy-Aware Approaches for Energy Harvesting Powered Wireless Sensor Nodes," in IEEE Sensors Journal, vol. 17, no. 7, pp. 2165-2173, 1 April1, 2017.

4. [17] D. Shen, J. Park, J. Ajitsaria, S. Choe, H C Wikle, and D Kim, "The design, fabrication and evaluation of a MEMS PZT cantilever with an integrated Si proof mass for vibration," J. Micromech. Microeng. vol. 18, no. 5, p. 055017, 2008.

5. [18] R. Ly, M. Rguiti, S. D. Astorg, A. Hajjaji, C. Courtois, and A. Leriche, "Physical Modeling and characterization of piezoelectric cantilever bending sensor for energy harvesting," Sensors and Actuators A . vol. 168, no. 1, pp. 95-100, 2011.
6. Tang, G., et al., Development of high performance piezoelectric d33 mode MEMS vibration energy harvester based on PMN-PT single crystal thick film. Sensors and Actuators A: Physical, 2014. 205: p. 150-155.

7. Aktakka, E.E., R.L. Peterson, and K. Najafi. A CMOS-compatible piezoelectric vibration energy scavenger based on the integration of bulk PZT films on silicon. in 2010 International Electron Devices Meeting. 2010. IEEE.

8. $\mathrm{Yi}$, Z., et al., High performance bimorph piezoelectric MEMS harvester via bulk PZT thick films on thin beryllium-bronze substrate. Applied Physics Letters, 2017. 111(1): p. 013902.

9. Kuo, C.-L., S.-C. Lin, and W.-J. Wu, Fabrication and performance evaluation of a metal-based bimorph piezoelectric MEMS generator for vibration energy harvesting. Smart Materials and Structures, 2016. 25(10): p. 105016.

10. Jackson, N., et al., Shock-induced aluminum nitride based MEMS energy harvester to power a leadless pacemaker. Sensors and Actuators A: Physical, 2017. 264: p. 212-218.

11. Wang, Z., et al., Self-Powered Viscosity and Pressure Sensing in Microfluidic Systems Based on the Piezoelectric Energy Harvesting of Flowing Droplets. ACS applied materials \& interfaces, 2017. 9(34): p. 28586-28595.

12. Tian, Y., et al., A low-frequency MEMS piezoelectric energy harvester with a rectangular hole based on bulk PZT film. Journal of Physics and Chemistry of Solids, 2018. 117: p. 21-27.

13. Won, S.S., et al., Flexible vibrational energy harvesting devices using strain-engineered perovskite piezoelectric thin films. Nano Energy, 2019. 55: p. 182-192.

14. H. Liu, C. J. Tay, C. Quan, T. Kobayashi, and C. Lee, "Piezoelectric MEMS energy harvester for low-frequency vibrations with wideband operation range and steadily increased output power," Microelectromechanical Syst. J., vol. 20, no. 5, pp. 1131-1142, 2011.

15. M. Rezaeisaray, M. El Gowini, D. Sameoto, D. Raboud, and W. Moussa, "Low frequency piezoelectric energy harvesting at multi vibration mode shapes," Sensors Actuators A Phys., vol. 228, pp. 104-111, 2015.

16. A. Hajati and S.-G. Kim, "Ultra-wide bandwidth piezoelectric energy harvesting," Appl. Phys. Lett., vol. 99, no. 8, p. 83105, 2011.

17. S. Leadenham and A. Erturk, "Nonlinear M-shaped broadband piezoelectric energy harvester for very low base accelerations: primary and secondary resonances," Smart Mater. Struct., vol. 24 no. 5, p. 55021, 2015.

18. D. S. Nguyen, E. Halvorsen, G. U. Jensen, and A. Vogl, "Fabrication and characterization of a wideband MEMS energy harvester utilizing nonlinear springs," J. Micromechanics Microengineering, vol. 20, no. 12, p. 125009, 2010.

Published By:

Blue Eyes Intelligence Engineering

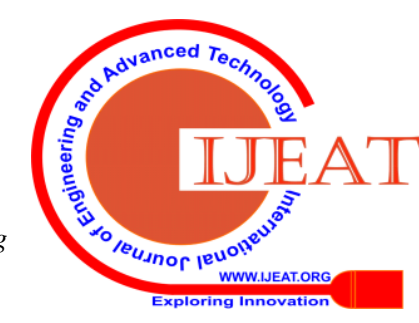


19. Y. Zhang, T. Wang, A. Luo, Y. Hu, X. Li, and F. Wang, "Micro electrostatic energy harvester with both broad bandwidth and high normalized power density,” Appl. Energy, vol. 212, pp. 362-371, 2018.

20. K. Tao, L. Tang, J. Wu, S. W. Lye, H. Chang, and J. Miao, "Investigation of multimodal electret-based MEMS energy harvester with impact-induced nonlinearity," J. Microelectromechanical Syst., vol. 27, no. 2, pp. 276-288, 2018.

21. H. Liu, Y. Qian, and C. Lee, "A multi-frequency vibration-based MEMS electromagnetic energy harvesting device," Sensors Actuators A Phys., vol. 204, pp. 37-43, 2013.

22. D. Mallick, A. Amann, and S. Roy, "A nonlinear stretching based electromagnetic energy harvester on FR4 for wideband operation," Smart Mater. Struct., vol. 24, no. 1, p. 15013, 2014.

23. N. Wang et al., "AIN wideband energy harvesters with wafer-level vacuum packaging utilizing three-wafer bonding," in Proc. IEEE Micro Electro Mechanical Systems (MEMS), 2017, pp. 841-844.

24. Y. Jia and A. A. Seshia, "Power optimization by mass tuning for MEMS piezoelectric cantilever vibration energy harvesting," $J$ Microelectromechanical Syst., vol. 25, no. 1, pp. 108-117, 2016.

25. Y. Jia, S. Du, E. Arroyo, and A. A. Seshia, "Autoparametric resonance in a piezoelectric MEMS vibration energy harvester," in Proc. IEEE Micro Electro Mechanical Systems (MEMS), 2018, pp. 226-229.

26. Y. Hu and Y. Xu, "A wideband vibration energy harvester based on a folded asymmetric gapped cantilever," Appl. Phys. Lett., vol. 104, no. 5, p. 53902, 2014.

27. E. E. Aktakka and K. Najafi, "Three-axis piezoelectric vibration energy harvester," in Proc. IEEE Micro Electro Mechanical Systems (MEMS), 2015, pp. 1141-1144.

28. H. Liu, C. Lee, T. Kobayashi, C. J. Tay, and C. Quan, "Piezoelectric MEMS-based wideband energy harvesting systems using a frequency-up-conversion cantilever stopper," Sensors Actuators A Phys., vol. 186, pp. 242-248, 2012.

29. S. Nabavi and L. Zhang, "Nonlinear Multi-Mode Wideband Piezoelectric MEMS Vibration Energy Harvester," in IEEE Sensors Journal, vol. 19, no. 13, pp. 4837-4848, 1 July1, 2019.

\section{AUTHORS PROFILE}

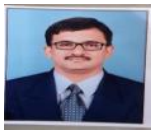

Mude Sreenivasulu, Research Scholar at JNTUH, Hyderabad and Associate Professor at Samskruti College of Engineering and Technology, Hyderabad, received Bachelors Degree (ECE) in 2001 from KSRM College of Engineering, S.V University, Tirupathi, and M.Tech (DECS)from MITS College, JNTU Hyderabad in 2006. He is having total fifteen years of teaching experience as an Assistant and Associate Professor in various Engineering Colleges in Andhra Pradesh, India Since 2002. Currently, he is working as an Associate Professor at Samskruti college of Engineering \&Technolog, Hyderabad, T.S since August 2019 and pursuing External Ph.D at JNT University, Hyderabad since 2011. He has published over 22 Papers at National \& Internationl conferences and in International Journals. His research interests include RF MEMS applications, Digital Electronics, Microelectronic devices and etc.

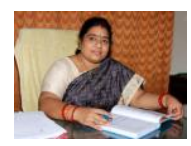

Dr. V. Usha Shree, Professor \& Principal of ECE dept, Joginpally B.R Engineering College, Hyderabad, has an experience of 20 years in teaching and research put together. She obtained her B.Tech (ECE), M.Tech (ECE) and Ph.D (ECE) from Jawaharlal Nehru Technological University, College of Engineering, Ananthapur. She obtained her Ph.D in the area of MEMS sensors and Embedded Systems. She is versatile in multidisciplinary specializations in allied branches. Her laurels include more than 109 publications in the National and International reputed conferences and journals. She is the fellow of IETE, Life member of ISTE. As a Chief coordinator of Industry Institute Partnership Cell (IIPC), her efforts of networking with industry for faculty and student development programs are appreciated. She worked as principal for leading Engineering College, and was responsible for establishing the facilities and nurturing the faculty and student to build managerial competencies.

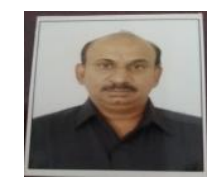

Dr. P. Chandra Sekhar Reddy, Professor of ECE, BOS Chairman, JNTUCE, JNTUH, Hyderabad has received his Bachelor of Technology Degree(ECE) from JNTUCE, Ananthapur .He has received double masters degress M.E in Applied Electronics from Bharatiar University and M.Tech from JNTU, Hyderabad. He was awarded Ph.D from JNTUH in 2000, Hyderabad in wireless Communications. He has got vast experience in teaching since 1992 as an Assistant Professor, Associate Professor and Professor since April 2006 at JNTUH, Hyderabad. He has published more than 130 journals at national and International levels and more than 120 papers at International and national level conferences.

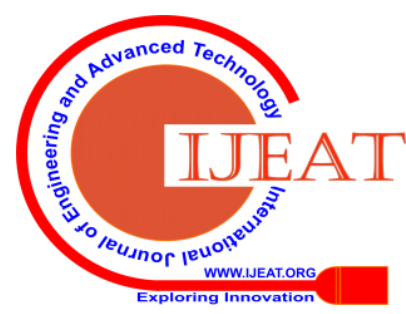

